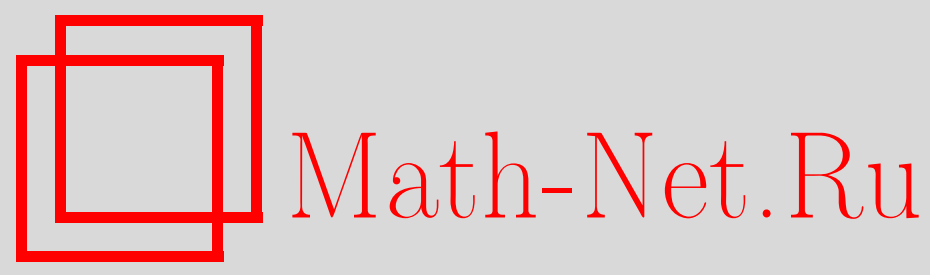

Н. А. Малаксиано, О точных вложениях классов Геринга в классы Макенхаупта, Матем. заметки, 2001, том 70, выпуск $5,742-750$

DOI: https://doi.org/10.4213/mzm785

Использование Общероссийского математического портала Math-Net.Ru подразумевает, что вы прочитали и согласны с пользовательским соглашением http://www . mathnet.ru/rus/agreement

Параметры загрузки:

IP : 18.234 .197 .8

26 апреля 2023 г., 11:54:18

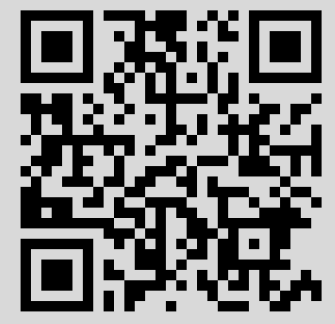




\section{О ТОЧНЫХ ВЛОЖЕНИЯХ КЛАССОВ ГЕРИНГА \\ В КЛАССЫ МАКЕНХАУПТА}

\section{Н. А. Малаксиано}

В одномерном случае находится точная граница показателей классов функций Макенхаупта, в которые вложен заданный класс Геринга.

Библиограбоия: 12 названий.

1. Введение. Будем считать все функции измеримыми и неотрицательными на кубе $Q_{0} \subset \mathbb{R}^{n}$ (рассматриваются только кубы со сторонами, параллельньми координатным осям). Обозначим через $|E|$ меру Лебега множества $E \subset Q_{0}$ и

$$
f_{E} \stackrel{\text { def }}{=} f_{E} f(x) d x \stackrel{\text { def }}{=} \frac{1}{|E|} \int_{E} f(x) d x .
$$

ОПРЕДЕЛЕнИЕ 1.1. Пусть $q>1$ и $B>1$. Говорят, что функция $f$ принадлежит классу Макенхаупта $A_{q}(B)$ на кубе $Q_{0} \subset \mathbb{R}^{n}$, если для любого подкуба $Q \subset Q_{0}$ имеет место неравенство

$$
\left(f_{Q} f(x) d x\right)\left(f_{Q} f^{-1 /(q-1)}(x) d x\right)^{q-1} \leqslant B
$$

Такие классы функций играют важную роль в теории весовых пространств и в ряде других вопросов. В работе Макенхаупта [1] доказано, что из принадлежности функции классу $A_{q}(B)$ следует ее принадлежность классу $A_{q-\varepsilon}\left(B_{1}\right)$ при некотором $\varepsilon>0$. Другими словами, имеет место включение

$$
A_{q}(B) \subset A_{q-\varepsilon}\left(B_{1}\right) .
$$

ОПРЕДЕЛЕНИЕ 1.2. Пусть $p>1$ и $C>1$. Говорят, что функция $f$ принадлежит классу Геринга $G_{p}(C)$ на кубе $Q_{0} \subset \mathbb{R}^{n}$, если для любого подкуба $Q \subset Q_{0}$ функция $f$ удовлетворяет "обратному неравенству Гёльдера" (условию Геринга):

$$
\left(f_{Q} f^{p}(x) d x\right)^{1 / p} \leqslant C\left(f_{Q} f(x) d x\right) .
$$

Герингом [2] было показано, что для любого класса $G_{p}(C)$ найдется $\varepsilon>0$, при котором имеет место включение

$$
G_{p}(C) \subset G_{p+\varepsilon}\left(C_{1}\right)
$$


Последний результат имеет многочисленные применения в теории весовых пространств, квазиконформных отображений, дифференциальньг уравнений с частными производными.

Имеется ряд работ, в которых исследуется связь между классами Геринга и Макенхаупта. Так, в работе Койфмана и Феффермана [3] доказано, что каждый класс Геринга содержится в некотором классе Макенхаупта и наоборот, т.е. имеют место включения вида

$$
A_{q}(B) \subset G_{p}(C)
$$

и

$$
G_{p_{1}}\left(C_{1}\right) \subset A_{q_{1}}\left(B_{1}\right) .
$$

В работе Боярского [4] для фиксированного класса $G_{p}(C)$ была поставлена задача нахождения предельного значения $\varepsilon=\varepsilon(n, p, C)$ во включении (1.2). Асимптотическая оценка такого $\varepsilon(n, p, C)$ при $C \rightarrow 1+$ получена Боярским [4], [5] и Виком [6]. В последнее время получены и другие асимптотические зависимости между параметрами классов Геринга и Макенхаупта (см., например, [7]).

В одномерном случае при решении поставленного вопроса удалось продвинуться дальше. Так, для монотонных функций Сбордоне и Д'Апуцо [8], [9] были получены точные гранищы показателей, при которьх имеют место вложения (1.2) для классов Геринга. Именно в [9] показано, что если невозрастающая на $[a, b]$ функция $f \in G_{p}(C)$, то для любого $p_{1}<p_{0}$ существует такое $C_{1}$, не зависящее от $f$, что $f \in G_{p_{1}}\left(C_{1}\right)$, где число $p_{0}>p$ определяется как корень уравнения

$$
\frac{p_{0}-p}{p_{0}}\left(\frac{C p_{0}}{p_{0}-1}\right)^{p}=1
$$

причем значение $p_{0}$, вообще говоря, нельзя увеличить.

Кореновским [10] последний результат был обобщен на случай произвольной функции одной переменной. Также в [10] находятся точные границы показателей, при которых в одномерном случае имеют место вложения (1.1) для классов Макенхаупта. Именно в [10] показано, что если функция $f \in A_{q}(B)$ на $[a, b]$, то для любого $q_{1}>q_{0}$ сушествует такое $B_{1}$, не зависящее от $f$, что $f \in A_{q_{1}}\left(B_{1}\right)$, где число $q_{0}>1$ определяется равенством

$$
\frac{q-q_{0}}{q-1}\left(B q_{0}\right)^{1 /(q-1)}=1
$$

причем значение $q_{0}$, вообще говоря, нельзя увеличить.

Таким образом, в одномерном случае решен вопрос нахождения точных гранищ показателей, при которых имеют место включения вида (1.1) и (1.2). Для включений вида (1.3) и (1.4) этот вопрос, насколько нам известно, оставался открытьп.

Целью данной работы является нахождения точных границ показателей, при которых имеют место включения вида (1.4). Главный результат представлен в следующей теореме.

ТЕОРема 1. Пусть $n=1, p>1 u C>1$, a $q_{0}>1$ определяется как корень уравнения

$$
q_{0} \cdot\left(\frac{1}{1+p\left(q_{0}-1\right)}\right)^{1 / p}=C
$$


Тогда для любого $q \in\left(q_{0},+\infty\right)$ существует $B=B(p, C, q)<\infty$ такое, ито имеет место включение

$$
G_{p}(C) \subset A_{q}(B),
$$

причем для $q \leqslant q_{0}$ әто включение неверно при любом $B$.

Для доказательства этой теоремы сначала рассматривается ее аналог для монотонных функций. Затем с помощью соответствующего неравенства для равноизмеримых перестановок (теорема A, п. 2) осуществляется переход от монотонных функций к произвольным. Такой переход применялся и ранее, например, Франциози и Москарелло [11] для доказательства вложений классов Геринга. Позднее, благодаря точным оценкам перестановок в [10] этот переход стал пригоден для получения точных показателей в (1.1) и (1.2) в одномерном случае.

Основным этапом в доказательстве теоремы 1 является получение ее аналога для монотонных функций (теорема $1^{\prime}$, п. 3). Тот факт, что точность предельных показателей для включений вида (1.1) и (1.2) может быть проверена на степенных функциях, навела на мысль о целесообразности выработки соответствующего подхода к доказательству теоремы $1^{\prime}$. Метод доказательства теоремы $1^{\prime}$ отличается от метода, использованного в работах [9] и [10], и заключается в сравнении монотонных функций со степенными.

Автор глубоко благодарен А. А. Кореновскому за постановку задачи, ценные советы и плодотворные обсуждения а также В. И. Коляде за внимание и полезные замечания, высказанные при подготовке данной работы к печати.

2. Вспомогательные результаты. Обозначим через $\Phi$ класс всех положительных на $[0,+\infty)$ вьпуклых вниз функций.

Лемма 2.1. Пусть функиии $f$ и не убъвают на $(a, b)$. Для того чтобы при любой $\varphi \in \Phi$ имело место неравенство

$$
\int_{a}^{b} \varphi(g(x)) d x \leqslant \int_{a}^{b} \varphi(f(x)) d x
$$

необходимо и достаточно, чтобы были выполнены следующие два условия:

$$
\int_{a}^{b} g(x) d x=\int_{a}^{b} f(x) d x
$$

$u$

$$
\int_{a}^{x} g(y) d y \geqslant \int_{a}^{x} f(y) d y \quad(a \leqslant x \leqslant b) .
$$

В работе [12] доказан аналог этой леммы для невозрастающих функций $f$ и $g$. Лемма 2.1 непосредственно следует из [12], если рассмотреть функции $g(a+b-x)$ и $f(a+b-x)$.

ОПРЕДЕЛЕНИЕ 2.1. Пусть $p>1$ и функция $f$ задана на интервале $(a, b)$. Будем обозначать

$$
G_{p}(f) \stackrel{\text { def }}{=} G_{p}(f ;(a, b)) \stackrel{\text { def }}{=} \sup _{I \subset(a, b)}\left(f_{I} f^{p}(x) d x\right)^{1 / p}\left(f_{I} f(x) d x\right)^{-1},
$$

где верхняя грань берется по всем подинтервалам $I \subset(a, b)$.

В дальнейшем нам понадобится следующее свойство параметров классов Геринга для степенных функций. 
ЛЕмма 2.2. Если $p>1 u \alpha>-1 / p, m o$

$$
G_{p}\left(x^{\alpha}\right) \equiv G_{p}\left(x^{\alpha} ;(0,1)\right)=\frac{1+\alpha}{(1+p \alpha)^{1 / p}} .
$$

ДокАЗАТЕЛЬСТво. По определению $G_{p}(f)$ имеем

$$
\begin{aligned}
G_{p}\left(x^{\alpha}\right) & =\sup _{(a, b) \subset(0,1)}\left(\frac{1}{b-a} \int_{a}^{b} x^{p \alpha} d x\right)^{1 / p}\left(\frac{1}{b-a} \int_{a}^{b} x^{\alpha} d x\right)^{-1} \\
& =\frac{1+\alpha}{(1+p \alpha)^{1 / p}} \cdot \sup _{0<a<b<1}(b-a)^{1-1 / p}\left(b^{p \alpha+1}-a^{p \alpha+1}\right)^{1 / p}\left(b^{\alpha+1}-a^{\alpha+1}\right)^{-1} .
\end{aligned}
$$

Обозначим $t \equiv b / a$ и запишем

$$
\begin{aligned}
& (b-a)^{1-1 / p}\left(b^{p \alpha+1}-a^{p \alpha+1}\right)^{1 / p}\left(b^{\alpha+1}-a^{\alpha+1}\right)^{-1} \\
& \quad=(t-1)^{1-1 / p}\left(t^{p \alpha+1}-1\right)^{1 / p}\left(t^{\alpha+1}-1\right)^{-1} \stackrel{\stackrel{\text { def }}{=}}{=}(t, p, \alpha),
\end{aligned}
$$

где $t>1, p>1$ и $\alpha>-1 / p$. Лемма будет доказана, если мы покажем, что

$$
\sup _{t>1} \zeta(t, p, \alpha)=1
$$

для любых фиксированных $p>1$ и $\alpha>-1 / p$.

Сначалапокажем, что $\zeta(t, p, \alpha) \leqslant 1$ для любых $t>1, p>1$ и $\alpha>-1 / p$. Зафиксируем $t>1, p>1$ и обозначим $v \equiv t^{\alpha}$. Тогда

$$
\zeta(t, p, \alpha) \equiv \xi(v) \stackrel{\text { def }}{=} \frac{\left(v^{p} t-1\right)^{1 / p}}{v t-1}(t-1)^{1-1 / p},
$$

где $v \in\left(t^{-1 / p},+\infty\right)$. Исследуя производную $\xi^{\prime}(v)$, при наших ограничениях легко видеть, что на промежутке $\left(t^{-1 / p},+\infty\right)$ функция $\xi(v)$ принимает максимальное значение в точке $v=1$, причем $\xi(1)=1$. Следовательно, $\zeta(t, p, \alpha) \leqslant 1$.

Ясно, что при фиксированных $p>1$ и $\alpha>-1 / p$ имеет место равенство

$$
\lim _{t \rightarrow+\infty} \zeta(t, p, \alpha)=1
$$

Из вышесказанного следует, что $\sup _{t>1} \zeta(t, p, \alpha)=1$.

Лемма доказана.

ЗАмЕчаниЕ 2.1. Из леммы 2.2 сразу следует, что если $0<\alpha<\beta$ и $p>1$, то $G_{p}\left(x^{\alpha} ;(0,1)\right)<G_{p}\left(x^{\beta} ;(0,1)\right)$.

Для изучения свойств функций часто бывает эффективным рассмотрение их равноизмеримых перестановок. Напомним определение.

ОПРЕДЕЛЕНИЕ 2.2. Невозрастающей перестановкой функции $f$ на промежутке $I$ назьвается функция

$$
f^{*}(t)=\sup _{\substack{e \subset I,|e| \geqslant t \\|e| \geqslant e}} \inf _{x \in e} f(x) \quad(0 \leqslant t \leqslant|I|) .
$$

Ниже нам понадобится следующая точная оценка перестановки. 
Tеорема А (см. [10]). Пусть $\varphi \in \Phi$ и функиия $f$ такая, что $\varphi(f) \in L[a, b]$. Тогда

$$
\sup _{I \subset(0, b-a)} \frac{f_{I} \varphi\left(f^{*}(t)\right) d t}{\varphi\left(f_{I} f^{*}(t) d t\right)} \leqslant \sup _{J \subset(a, b)} \frac{f_{J} \varphi(f(x)) d x}{\varphi\left(f_{J} f(x) d x\right)}
$$

где верхние грани берутся по всем подинтервалам соответствующих интервалов.

3. Доказательство теоремы 1. Как уже отмечалось во введении, сначала (см. ниже теорему 1') мы докажем аналог теоремы 1 для неубывающих функций, а затем обобщим его на случай произвольных функций одной переменной.

Для доказательства теоремы $1^{\prime}$ нам понадобится следующая

Лемма 3.1. Пусть числа $p>1 u 0<\tau<\alpha<+\infty$. Тогда существует $\varepsilon=\varepsilon(\alpha, \tau)>0$ такое, что для любой неубивающей на $(0,1)$ функиии $f$, удовлетворяющей условиям

$$
G_{p}(f ;(0,1)) \leqslant G_{p}\left(x^{\tau} ;(0,1)\right) \quad u \quad \int_{0}^{1} f(x) d x \geqslant 2 \int_{0}^{1} x^{\tau} d x
$$

имеет место неравенство $f(x) \geqslant x^{\alpha}, 0<x<\varepsilon$.

ДокАЗАТЕЛЬСтво. Доказательство проведем от противного. Предположим, что утверждение леммы неверно, т.е. предположим, что имеет место следующее

УТВЕРЖДЕНИЕ А. Для любого $\varepsilon>0$ существует неубывающая на $(0,1)$ функиия $f$, для которой

$$
\begin{gathered}
G_{p}(f ;(0,1)) \leqslant G_{p}\left(x^{\tau} ;(0,1)\right) \\
\int_{0}^{1} f(x) d x \geqslant 2 \int_{0}^{1} x^{\tau} d x
\end{gathered}
$$

и существует $a \in(0, \varepsilon)$ такое, что $f(a)<a^{\alpha}$.

Положим $\beta=(\alpha+\tau) / 2$.

Очевидно, для любого $h<1 / 3$ верно неравенство

$$
h^{\beta+1}<\frac{1}{2} \int_{h}^{1} x^{\beta} d x
$$

Возьмем $\varepsilon<1 / 3$, и пусть $f$ и $a-$ произвольные функция и число из утверждения А c выбранным $\varepsilon$.

Возьмем точку $h_{1}=a^{\alpha / \beta}$.

Заметим, что для любого $x \in\left[h_{1}, a\right]$ верно $f(x)<x^{\beta}$. Действительно, при $x \in\left[h_{1}, a\right]$ имеем

$$
f(x) \leqslant f(a)<a^{\alpha}=\left(h_{1}^{\beta / \alpha}\right)^{\alpha}=h_{1}^{\beta} \leqslant x^{\beta} .
$$


Так как $h_{1}<a<\varepsilon<1 / 3$, то, в силу (3.3) верно неравенство

$$
\int_{0}^{h_{1}} f(x) d x \leqslant h_{1}^{\beta+1}<\frac{1}{2} \int_{h_{1}}^{1} x^{\beta} d x .
$$

На промежутке $\left[h_{1}, 1\right]$ рассмотрим функцию

$$
F(x) \stackrel{\text { def }}{=} \int_{h_{1}}^{x} f(y) d y-\int_{h_{1}}^{x} y^{\beta} d y .
$$

Очевидно, $F$ непрерьвна, $F\left(h_{1}\right)=0$ и при $x \in\left(h_{1}, a\right)$ имеет место неравенство $F(x)<0$. Определим знак $F(1)$. Учитьвая (3.4) и (3.2), имеем:

$$
\begin{aligned}
F(1) & =\int_{h_{1}}^{1} f(y) d y-\int_{h_{1}}^{1} y^{\beta} d y=\int_{h_{1}}^{1} f(y) d y+\frac{1}{2} \int_{h_{1}}^{1} y^{\beta} d y-\frac{3}{2} \int_{h_{1}}^{1} y^{\beta} d y \\
& >\int_{h_{1}}^{1} f(y) d y+\int_{0}^{h_{1}} f(y) d y-\frac{3}{2} \int_{h_{1}}^{1} y^{\beta} d y=\int_{0}^{1} f(y) d y-\frac{3}{2} \int_{h_{1}}^{1} y^{\beta} d y \\
& \geqslant 2 \int_{0}^{1} y^{\tau} d y-\frac{3}{2} \int_{h_{1}}^{1} y^{\beta} d y>0 .
\end{aligned}
$$

Из перечисленных свойств функции $F$ следует существование $h_{2} \in(a, 1)$ такого, что $F\left(h_{2}\right)=0$ и для любого $x \in\left(h_{1}, h_{2}\right)$ верно неравенство $F(x)<0$.

Таким образом, для функций $f$ и $x^{\beta}$ на промежутке $\left[h_{1}, h_{2}\right]$ вьполнены все условия леммы 2.1. Взяв в условии леммы $2.1 \varphi(s)=s^{p}$, получим

$$
\int_{h_{1}}^{h_{2}} f^{p}(x) d x \geqslant \int_{h_{1}}^{h_{2}} x^{p \beta} d x .
$$

Заметим, что

$$
\frac{h_{2}}{h_{1}}>\frac{a}{h_{1}}=\frac{a}{a^{\alpha / \beta}}=a^{1-\alpha / \beta}>\varepsilon^{1-\alpha / \beta} .
$$

Подводя итог вышесказанному, получаем: для любого $\varepsilon<1 / 3$ и для любой функции $f$ из утверждения А с выбранным $\varepsilon$ существуют $h_{1}$ и $h_{2}$ из $(0,1)$ такие, что $h_{2} / h_{1}>$ $\varepsilon^{1-\alpha / \beta}$, и имеет место неравенство

$$
\begin{aligned}
G_{p}(f) & \geqslant\left(f_{h_{1}}^{h_{2}} f^{p}(x) d x\right)^{1 / p}\left(f_{h_{1}}^{h_{2}} f(x) d x\right)^{-1} \geqslant\left(f_{h_{1}}^{h_{2}} x^{p \beta}(x) d x\right)^{1 / p}\left(f_{h_{1}}^{h_{2}} x^{\beta} d x\right)^{-1} \\
= & {\left[\frac{1}{1+p \beta}\left(h_{2}^{p \beta+1}-h_{1}^{p \beta+1}\right) \frac{1}{h_{2}-h_{1}}\right]^{1 / p}\left[\frac{1}{1+\beta}\left(h_{2}^{1+\beta}-h_{1}^{1+\beta}\right) \frac{1}{h_{2}-h_{1}}\right]^{-1} } \\
= & {\left[\frac{1}{1+p \beta}\left(\left(\frac{h_{2}}{h_{1}}\right)^{p \beta+1}-1\right) \frac{1}{h_{2} / h_{1}-1}\right)^{1 / p}\left[\frac{1}{1+\beta}\left(\left(\frac{h_{2}}{h_{1}}\right)^{1+\beta}-1\right) \frac{1}{h_{2} / h_{1}-1}\right]^{-1} . }
\end{aligned}
$$

Если будем выбирать $\varepsilon$ достаточно малым, то в силу неравенства (3.5) правая часть (3.6) будет сколь угодно мало отличаться от

$$
\left(\frac{1}{1+p \beta}\right)^{1 / p}\left(\frac{1}{1+\beta}\right)^{-1} .
$$


Но в силу леммы 2.2 и замечания 2.1

$$
\left(\frac{1}{1+p \beta}\right)^{1 / p}\left(\frac{1}{1+\beta}\right)^{-1}=G_{p}\left(x^{\beta}\right)>G_{p}\left(x^{\tau}\right)
$$

Значит, выбирая $\varepsilon$ достаточно малым, мы получим, что для любой функции $f$, удовлетворяющей утверждению А, из (3.6) будет следовать неравенство $G_{p}(f)>G_{p}\left(x^{\tau}\right)$, противоречащее (3.1).

Полученное противоречие доказьвает лемму.

ОПРЕДЕЛЕНИЕ 3.1. Обозначим $A_{q}^{*}(B)$ множество всех невозрастающих функций из $A_{q}(B)$, а через $G_{p}^{*}(C)$ - множество всех невозрастающих функций из $G_{p}(C)$.

Если в дальнейшем будет необходимо уточнить множество $Q$, на котором рассматриваются классы $A_{q}(B)$ или $G_{p}(C)$, мы будем использовать обозначения

$$
A_{q}(B ; Q) \equiv A_{q}(B) \quad \text { и } \quad G_{p}(C ; Q) \equiv G_{p}(C) .
$$

Teоpema $1^{\prime}$. Пусть $p \in(1,+\infty) u C>1$. Тогда

1) уравнение

$$
C=q_{0}\left(\frac{1}{1+p\left(q_{0}-1\right)}\right)^{1 / p}
$$

имеет единственный корень $q_{0}>1$;

2) для любого $q>q_{0}$ существует $B=B(p, C, q)<\infty$ такое, что для любого интервала $(a, b)$

$$
G_{p}^{*}(C ;(a, b)) \subset A_{q}^{*}(B ;(a, b)) ;
$$

3) при $q \leqslant q_{0}$ утверждение 2) теряет силу.

ДокАЗАТЕЛЬСтво. Докажем каждое утверждение теоремы.

1) Следует из того, что правая часть (3.7) непрерьвна как функция $q_{0}$ и строго возрастает от 1 до $+\infty$ при $q_{0}>1$.

2) Зафиксируем $q>q_{0}$ и покажем, что если произвольная невозрастающая на $(a, b)$ функция $f \in G_{p}(C)$, то $f \in A_{q}(B)$, где $B$ - некоторая постоянная, не зависящая от $f$ и $(a, b)$.

Обозначим $q_{1}=\left(q+q_{0}\right) / 2$.

Возьмем произвольный интервал $\left(h_{1}, h_{2}\right) \subset(a, b)$.

Обозначим $g_{1}(x) \stackrel{\text { def }}{=} f\left(x\left(h_{1}-h_{2}\right)+h_{2}\right)$ и рассмотрим

$$
g(x) \stackrel{\text { def }}{=} \frac{2 \int_{0}^{1} y^{q_{0}-1} d y}{\int_{0}^{1} g_{1}(y) d y} \cdot g_{1}(x), \quad 0<x<1 .
$$

Легко проверить, что $g$ не убывает на $(0,1)$ и

$$
\left(f_{h_{1}}^{h_{2}} f(x) d x\right)\left(f_{h_{1}}^{h_{2}} f^{-1 /(q-1)}(x) d x\right)^{q-1}=\left(\int_{0}^{1} g(x) d x\right)\left(\int_{0}^{1} g^{-1 /(q-1)}(x) d x\right)^{q-1} .
$$


Очевидно,

$$
G_{p}(g ;(0,1))=G_{p}\left(f ;\left(h_{1}, h_{2}\right)\right) \leqslant G_{p}(f ;(a, b)) \leqslant C .
$$

Далее, по лемме 2.2 имеем

$$
C=q_{0}\left(\frac{1}{1+p\left(q_{0}-1\right)}\right)^{1 / p}=G_{p}\left(x^{q_{0}-1} ;(0,1)\right) .
$$

Поэтому $G_{p}(g ;(0,1)) \leqslant G_{p}\left(x^{q_{0}-1} ;(0,1)\right)$.

Непосредственно из определения функции $g$ следует, что

$$
\int_{0}^{1} g(x) d x=2 \int_{0}^{1} x^{q_{0}-1} d x .
$$

Таким образом, для чисел $p$ и $q_{0}-1<q_{1}-1$ и функции $g$ вьполнены условия леммы 3.1. В силу леммы 3.1 существует $\varepsilon=\varepsilon\left(q_{0}, q_{1}\right)$, не зависящее от функиии $g$, такое, что $g(x) \geqslant x^{q_{1}-1}, 0<x<\varepsilon$. Отсюда

$$
\int_{0}^{1} g^{-1 /(q-1)}(x) d x \leqslant \int_{0}^{\varepsilon} x^{-\left(q_{1}-1\right) /(q-1)} d x+(1-\varepsilon) \varepsilon^{-\left(q_{1}-1\right) /(q-1)}<+\infty .
$$

Подставляя (3.10) и (3.9) в (3.8), получим

$$
\begin{aligned}
& \left(f_{h_{1}}^{h_{2}} f(x) d x\right)\left(f_{h_{1}}^{h_{2}} f^{-1 /(q-1)}(x) d x\right)^{q-1}=\left(\int_{0}^{1} g(x) d x\right)\left(\int_{0}^{1} g^{-1 /(q-1)}(x) d x\right)^{q-1} \\
& \quad \leqslant\left(2 \int_{0}^{1} x^{q_{0}-1} d x\right)\left(\int_{0}^{\varepsilon} x^{-\left(q_{1}-1\right) /(q-1)} d x+(1-\varepsilon) \varepsilon^{-\left(q_{1}-1\right) /(q-1)}\right)^{q-1} \cdot
\end{aligned}
$$

Заметим, что правая часть (3.11) не зависит от функции $f$ и выбора промежутков $(a, b)$ и $\left(h_{1}, h_{2}\right)$, а зависит только от $q_{0}, q_{1}, q$ и $\varepsilon$, т.е. от $p, C$ и $q$. Обозначим правую часть $(3.11)$ через $B \equiv B(p, C, q)$. Таким образом, мы получили, что для любого промежутка $\left(h_{1}, h_{2}\right)$ $\subset(a, b)$ имеет место неравенство

$$
\left(f_{h_{1}}^{h_{2}} f(x) d x\right)\left(f_{h_{1}}^{h_{2}} f^{-1 /(q-1)}(x) d x\right)^{q-1} \leqslant B
$$

что доказьвает утверждение 2).

3) Покажем, что для любых $p>1$ и $C>1$ в классе $G_{p}(C ;(0,1))$ существует такая функция $f$, которая не только не принадлежит никакому классу $A_{q}(B ;(0,1))$, где $q \leqslant q_{0}$ и $q_{0}$ - положительньй корень уравнения (3.7), но даже не суммируема со степенью $-1 /\left(q_{0}-1\right)$.

Действительно, зафиксируем произвольные $p>1$ и $C>1$. Пусть $q_{0}=q_{0}(p, C)-$ положительньй корень уравнения (3.7). Возьмем $f(x)=x^{q_{0}-1}$. Ясно, что $f^{-1 /\left(q_{0}-1\right)}$ не суммируема на $(0,1)$. Покажем, что $f(x) \in G_{p}(C)$. По лемме 2.2

$$
G_{p}(f ;(0,1))=q_{0}\left(\frac{1}{1+p\left(q_{0}-1\right)}\right)^{1 / p}=C ;
$$

значит, $f \in G_{p}(C ;(0,1))$.

Теорема доказана полностью. 
ДокАЗАТЕЛЬСТво тЕОРЕМЫ 1. Для доказательства теоремы 1 нам осталось показать, что в утверждении 2) теоремы $1^{\prime}$ вместо включения $G_{p}^{*}(C) \subset A_{q}^{*}(B)$ можно гарантировать $G_{p}(C) \subset A_{q}(B)$.

Зафиксируем произвольную функцию $f \in G_{p}(C ;(a, b))$ и возьмем произвольньй интервал $I \subset(a, b)$. Рассмотрим сужение $f \mid I$. В силу теоремы А $(f \mid I)^{*} \in G_{p}(C ;(0,|I|))$. Применяя к перестановке $(f \mid I)^{*}$ теорему $1^{\prime}$, получим, что для любого $q>q_{0}$ существует $B=B(p, q, C)$ такое, что $(f \mid I)^{*} \in A_{q}(B ;(0,|I|))$. Значит,

$$
\begin{aligned}
& \left(f_{I} f(x) d x\right)\left(f_{I} f^{-1 /(q-1)}(x) d x\right)^{q-1} \\
& \quad=\left(f_{0}^{|I|}(f \mid I)^{*}(t) d t\right)\left(f_{0}^{|I|}\left((f \mid I)^{*}\right)^{-1 /(q-1)}(t) d t\right)^{q-1} \leqslant B .
\end{aligned}
$$

Так как $I \subset(a, b)$ - произвольньй интервал, то отсюда получаем, что $f \in A_{q}(B ;(a, b))$.

В силу произвольности выбора функции $f \in G_{p}(C ;(a, b))$ имеем $G_{p}(C ;(a, b)) \subset$ $A_{q}(B ;(a, b))$.

Теорема доказана.

\section{СПИСОК ЦИТИРОВАННОЙ ЛИТЕРАТУРЫ}

[1] Muckenhoupt B. Weighted norm inequalities for the Hardy maximal function // Trans. Amer. Math. Soc. 1972. V. 165. P. 207-226.

[2] Gehring F. W. The $L^{p}$-integrability of the partial derivatives of a quasiconformal mapping // Acta Math. 1973. V. 130. P. 265-277.

[3] Coifman R.R., Fefferman C. Weighted norm inequalities for maximal functions and singular integrals // Studia Math. 1974. V. 51. №3. P. 241-250.

[4] Bojarski B. Remarks on the stability of reverse Hölder inequalities and quasiconformal mappings // Ann. Acad. Sci. Fenn. A. Math. 1985. V. 10. P. 291-296.

[5] Bojarski B. On Gurov-Reshetnyak classes // Proc. BMO-Seminar (Joensu, Finland, June 1988): Joensu Univ., 1989.

[6] Wik I. Reverse Hölder inequalities with constant close to 1 // Ricerche di Matematica. 1990. V. 39. № 1. P. 151-157.

[7] Korey M. B. Ideal weights: asymptotically optimal versions of doubling, absolute continuity, and bounded mean oscillation // J. Fourier Anal. Appl. 1998. V. 4. № 4, 5. P. 491-519.

[8] Sbordone C. Rearrangement of functions and reverse Hölder inequalities // Research Notes in Math. 1983. V. 125. P. 139-148.

[9] D'Apuzzo L., Sbordone C. Reverse Hölder inequalities. A sharp result // Rendiconti di Math. Ser. VII. 1990. V. 10. P. 357-366.

[10] Кореновский А. А. О точном продолжении обратного неравенства Гёльдера и условия Макенхаупта // Матем. заметки. 1992. Т. 52. №6. С. 32-44.

[11] Franciosi M., Moscariello G. Higher integrability results // Manuscripta Math. 1985. V. 52. №1-3. P. 151-170.

[12] Hardy G. H., Littlewood J. E., Polya G. Some simple inequalities satisfied by convex functions // Messenger of Math. 1929. V. 58. P. 145-152. 